\title{
Weld Inspection Based on Radiography Image Segmentation with Level Set Active Contour Guided Off-Center Saliency Map
}

\author{
Mohamed Ben Gharsallah and Ezzeddine Ben Braiek \\ University of Tunis, Tunis National Higher School of Engineering (ENSIT), Research CEREP Unit, \\ 5 Avenue Taha Hussein, 1008 Tunis, Tunisia \\ Correspondence should be addressed to Mohamed Ben Gharsallah; medgharsallah@yahoo.fr
}

Received 7 September 2015; Revised 28 November 2015; Accepted 30 November 2015

Academic Editor: Ying Li

Copyright ( $) 2015$ M. Ben Gharsallah and E. Ben Braiek. This is an open access article distributed under the Creative Commons Attribution License, which permits unrestricted use, distribution, and reproduction in any medium, provided the original work is properly cited.

\begin{abstract}
Radiography is one of the most used techniques in weld defect inspection. Weld defect detection becomes a complex task when uneven illumination and low contrast characterize radiographic images. In this paper we propose a new active contour based level set method for weld defect detection in radiography images. An off-center saliency map exploited as a feature to represent image pixels is embedded into a region energy minimization function to guide the level set active contour to defects boundaries. The aim behind using salient feature is that a small defect can frequently attract attention of human eyes which permits enhancing defects in low contrasted image. Experiment results on different weld radiographic images with various kinds of defects show robustness and good performance of the proposed approach comparing with other segmentation methods.
\end{abstract}

\section{Introduction}

Nondestructive testing (NDT) is widely used in many fields, principally for serious applications where malfunction can be catastrophic such as welds of pressure vessels, aircraft, and power plants. One of the most famous techniques used in weld inspection is radiography which is based on the transmission of X-rays or gamma rays through an object to generate a radiological image on a photographic plate (Figure 1). Unfortunately, the traditional interpretation of radiography images by artificial methods is subjective, timeconsuming, and easy to cause fatigue, in order to improve the automation level and avoid drawbacks of manual interpretation; it is desirable to develop some forms of computer-aided systems to assist the human interpreter in evaluating the quality of welded joints. In general, this system of automatic inspection should have the following stages $[1,2]$; after digital image acquisition only a region of interest (ROI) is further processed, some preprocessing may take place like noise reduction and contrast enhancement and then segmentation of regions that may represent defects is done; as soon as the defects are segmented features can be extracted and then given as input to classifiers to detect possible defects and eventually to identify the exact defect type. Moreover the defect dimensions are compared to some acceptance criteria defined by experts or international standards and a decision is taken on the acceptability of the monitored weld.

As shown in Figure 2, weld radiography image contains two main parts: the base metal part and the weld seam part. The weld region is brighter than the weld area. Defects are randomly found at the weld area with different small shapes: circular and rectangular. Weld flaws can be categorized in various types like incomplete penetration, slag line, slag inclusion, cracks, undercuts, porosity, and wormholes. Porosity or gas cavity has rounded contours and dark shadows, cracks are fine line straight or wandering in direction, slag are line more or less interrupted parallel to the edges of weld. Radiography images are characterized by a low contrast between defects and background (weld) and small defects with blurred and unsharpened edges. Moreover, uneven illumination is frequently found in radiography images which is a nonuniform light distributed generally at the middle of weld area. For weld inspectors, these factors make defect localisation and segmentation with conventional segmentation methods a complicated mission. To overcome these difficulties and to facilitate human weld inspection, we 


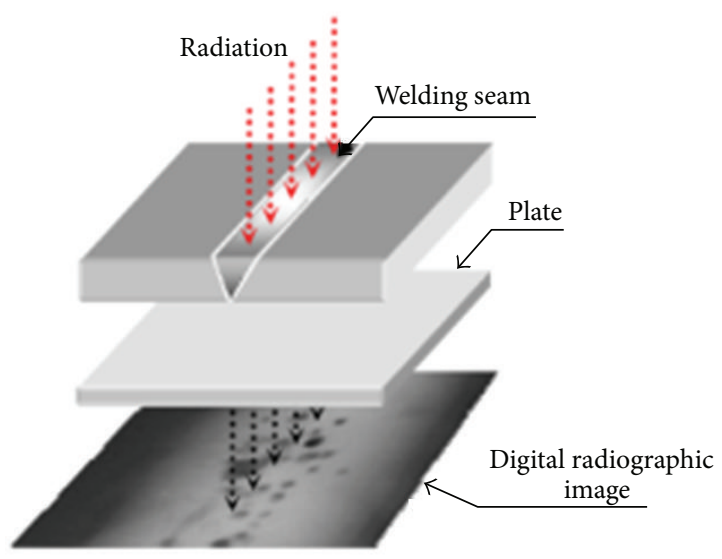

FIGURE 1: Weld radiography image acquisition [6].

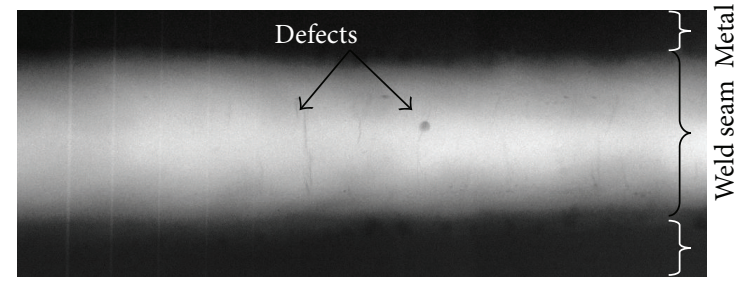

FIGURE 2: Example of weld radiography image with defects: porosities (circular) and a longitudinal crack.

propose in this paper an image segmentation method for radiography weld defect extraction.

In literature many radiography weld defect detection methods are proposed [3]. We find local and global thresholding approaches [4], texture analysis based methods [5], watershed [6], artificial neural network [7, 8], and active contours [9]. Segmentation with deformable models or active contours seems to be quite suitable for radiographic images to extract defects because of many reasons, principally the ability of integration of various image properties such as edge and region information with minimisation methods and curve theory. Several research works have been explored and many active contour models are proposed. In general active contour models can be categorized into two different classes: edge and region based models. Edge based models [10, 11] uses the edge information like image gradient to drive the active contour toward the object boundaries and to stop it there; these kinds of models are sensitive to noise and to initial active contour position which should be initialized near object boundaries. Moreover, the boundary leakage problem at weak edges is a major drawback of edge based models since they rely much on the gradient value. Compared with edge based models, region based models depend on statistical information inside and outside of regions delimited by the contour; thus they are less sensitive to the noise and to poor edges. Moreover, they are less dependent to the initialization since they exploit the global region information of the image statistics.
One of the most popular region based approaches is the Chan and Vese model well known as C-V [12]. C$\mathrm{V}$ model energy function is a simplification of Mumford Shah formulation [13]. It has been successfully applied in many applications for images with two regions; this model is less sensitive to image noise and contour initialization which can be located everywhere in the image. However, the major weakness of $\mathrm{C}-\mathrm{V}$ model is the segmentation of images with intensity inhomogeneities where pixels of the same object have a nonuniform gray level intensity distribution. To resolve this problem, active contours with localized energy functions have been proposed. The idea is to compute statistical information only in a local window in the pixel neighbourhood. One of the famous local region based active contours is proposed by Wang et al. [14, 15] called the local binary fitting (LBF) model. In this method two fitting energies are used to calculate LBF energy function that approximates the local image intensities means inside and outside of the contour using a Gaussian kernel. The LBF model solved the problem caused by intensity inhomogeneity. However, this model is sensitive to initial contour location and it increases greatly the computational complexity. Zhang et al. [16] proposed a selective local global level set active contour known as IVC model which introduces a new region signed pressure force (SPF) function. Zhang model uses a Gaussian smoothing kernel to regularize the level set function which decreases significantly the computation time. The main advantage of this model is the ability to select local or global segmentation so the user can choose to segment one object or the whole image. Nevertheless, experiments show that this model is very sensitive to nonuniform illumination and to low contrasted images like radiography images. Zhang et al. [17] proposed also a region local level set active contour where a local image fitting (LIF) energy function is used to guide the level set active contour to object boundaries based on local statistical information of the image. LIF energy function determines differences between the fitted image in the LBF model and the original image. In addition, a filtering method with a Gaussian kernel is applied to regularize level set function iteratively. LIF active contour model is well consuming computation time besides it is very sensitive to the local window sizes which should be adjusted carefully: choosing a small window size around pixel permits detection of small objects but increases sensitivity to noise. Song and Yan [18] proposed a local level set active contour to segment small defects found in hot rolled steel coloured images. The main idea is to fuse an image feature called the saliency map in the active contour energy formulation. This approach shows high performance in segmentation of defects with small regions particularly with rounded shapes in coloured images. Nevertheless, the saliency map used is adapted to coloured images so three information channels are needed. Much information might be missed when using images with one channel like radiography images. Moreover, Song model uses a Gaussian filter in the computation of the saliency map which is not suitable for images with low contrast images. In the other side, this approach is a level set local based model which increases the computation time considerably; besides 
segmentation accuracy depends greatly to the level set local window sizes.

In this work we propose a new global level set active contour to segment weld defects in radiography images. An image feature called the off-center saliency map computed with integral images is embedded in the energy formulation of a global region level set active contour. The remainder of this paper is organized as follows: the proposed method is presented in Section 2, tests and experimental results are depicted in Section 3, and we finish the paper by a conclusion in Section 4.

\section{Proposed Level Set Active Contour Model}

In this section we present the modified level set active contour. We show the off-center saliency map extraction method in the first part and the new level set active contour energy formulation after fusion with the extracted saliency map feature is illustrated in the second part.

2.1. Off-Center Saliency Map Computation. Systems that mimic the biological attention system are widely developed to extract the saliency map which aims to select the interesting parts of the sensory input data in order to reduce the vast amount of information that a computer vision system normally needs to process. In this area two types of retinal ganglion cells are defined: "on-center" and "off-center." An on-center cell is stimulated when the center of its receptive field is exposed to light and is inhibited when the surround is exposed to light; off-center cells have just the opposite reaction. Two image features that imitate the behavior of the two cells are computed and known as on-center and offcenter saliency maps. In weld radiography images, defects correspond to dark regions surrounded by brighten background; therefore, we decide to use the off-center saliency map as an image feature to guide the level set active contour to defect boundaries. Off-center saliency map is calculated using center-surround difference. Many approaches are used to achieve computation; we find Gabor filters [19], difference of Gaussian filters [20], and spectral residual [21]. Recently, Montabone and Soto [22] present a fast method to compute center-surround differences with rectangular filters based on the concept of integral images (or summed area tables) introduced first by Viola and Jones [23]; this approach allows speeding up the calculations considerably and preserving objects borders. This approach is used in our work. The offcenter saliency submaps are calculated by a difference center and surround with this relation:

$$
S_{c}(x, y)=\max \{\operatorname{surround}(x, y, c)-\operatorname{center}(x, y), 0\},
$$

where " $c$ " represents the surround size chosen empirically for our application as $c=\{2,4,8,16\}$ and center $(x, y)$ represents the gray level pixel at the surround middle $I(x, y)$.

The surround is calculated in a fast time using integral image $I_{g}(x, y)$ of the image $I(x, y)$ :

$$
I_{g}(x, y)=\sum_{i=0}^{x} \sum_{j=0}^{y} I(i, j) \text {. }
$$

The surround is the local average in neighbourhood window size " $c$ ":

$$
\text { surround }(x, y, c)=\frac{I_{g}(x+c, y+c)-I_{g}(x-c, y+c)-I_{g}(x+c, y-c)+I_{g}(x-c, y-c)-I(x, y)}{(2 c+1)^{2}-1}
$$

The computed off-center saliency submaps are summed pixel by pixel in a one map $S(x, y)$ as follows:

$$
S(x, y)=\sum_{c} S_{c}(x, y)
$$

$S(x, y)$ is the off-center saliency map which will be used in a further step with the level set active contour. The advantage of the feature $S(x, y)$ in defect enhancement is demonstrated by an example on radiographic weld image in Figure 3. As shown the weld radiography image has a low level contrast and a background affected by uneven illumination; defects and background are not easily distinguishable; therefore, defect extraction is very difficult. Figure 3(b) shows the offcenter saliency map $S(x, y)$, as we can see the difference between the defects and the background is highly enhanced; the surface plot of $S(x, y)$ shown in Figure 3(c) shows also how defects gray level are well amplified.
2.2. Level Set Active Contour Energy Formulation. The offcenter saliency map $S(x, y)$ obtained in the previous section is used as statistical information representing pixels in the image. The feature image $S(x, y)$ is embedded in the formulation of a level set active contour using a global Gaussian distribution fitting energy. The aim is to segment image into two regions, defect and background $\Omega_{1}, \Omega_{2}$, with a contour " $C$ " separating these two regions depending on statistical information inside and outside of the off-center saliency map $S(x, y)$. The segmentation is achieved with energy minimization [19]. We define an energy function to the contour " $C$ " composed of three terms: the salient energy $E_{1}(C)$, the regularization energy $E_{2}(C)$, and $E_{3}(C)$ the reinitialization energy:

$$
E(C)=E_{1}(C)+E_{2}(C)+E_{3}(C) .
$$




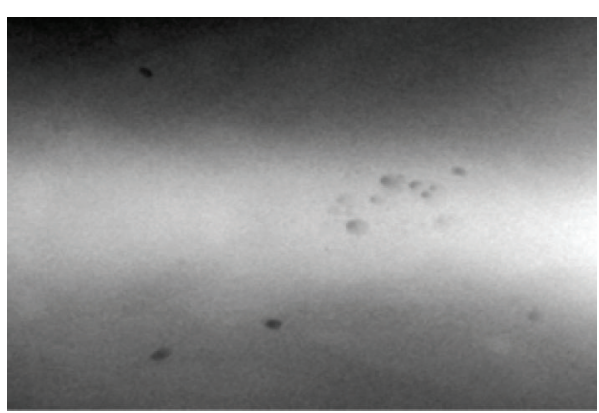

(a)

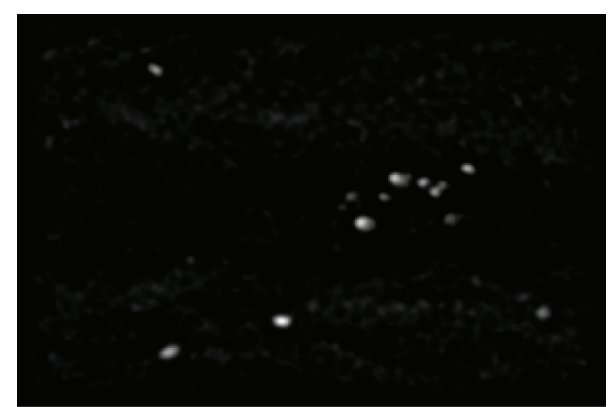

(b)

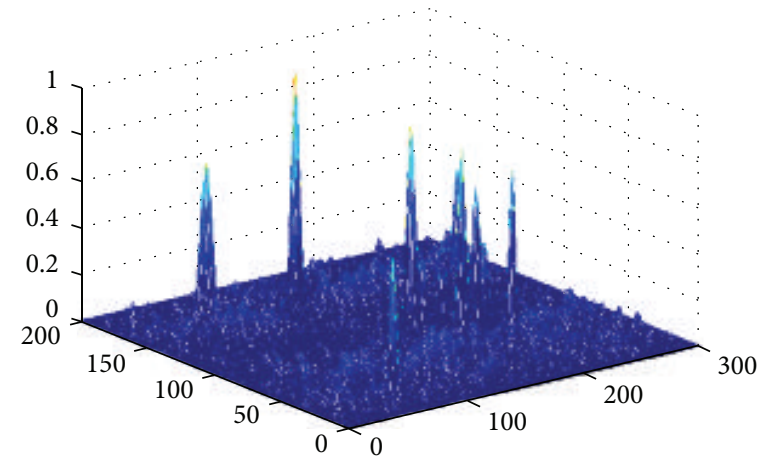

(c)

FIGURE 3: Example of off-center saliency map: (a) ROI radiography weld image, (b) off-center saliency map $S(x, y)$, and (c) surface plot of off-center saliency map.

The first term $E_{1}(C)$ attracts the contour " $C$ " to defect edges. We choose to minimize the Bayes error [24] defined as follows:

$$
\begin{aligned}
E_{1}(S(x, y), C)= & -\int_{\Omega_{1}} \log \left(P_{1}\left(S(x, y), \Omega_{1}\right)\right) d x d y \\
& -\int_{\Omega_{2}} \log \left(P_{2}\left(S(x, y), \Omega_{2}\right)\right) d x d y,
\end{aligned}
$$

where $\Omega_{1}, \Omega_{2}$ are the regions inside and outside the contour C. $P_{1}, P_{2}$ are two probability density functions (PDF). $S(x, y)$ is the off-center saliency map.

Based on the level set theory [16], the contour $C$ is embedded as the zero level of a level set function $\phi(x, y)$ :

$$
C=\{(x, y) \in \Omega: \phi(x, y)=0\}
$$

Thus (6) becomes

$$
\begin{aligned}
E_{1}( & (x, y), \phi(x, y)) \\
= & -\int_{\Omega} \log \left(P_{1}(S(x, y)) H(\phi(x, y))\right) d x d y \\
& -\int_{\Omega} \log \left(P_{2}(S(x, y))(1-H(\phi(x, y)))\right) d x d y,
\end{aligned}
$$

where $\Omega$ is the image domain and $H(\phi)$ is the Heaviside function.

$P_{1}, P_{2}$ are two probability density functions assumed as a Gaussian distribution with means $m_{1}, m_{2}$ and variances $\sigma_{1}$, $\sigma_{2}$. Consider

$$
P_{i}(S(x, y))=\frac{1}{\sqrt{2 \pi} \sigma_{i}} \exp \left(-\frac{\left(S(x, y)-m_{i}\right)^{2}}{2 \sigma_{i}^{2}}\right),
$$

$$
i=1,2 \text {. }
$$

Therefore (8) can be written as

$$
E_{1}\left(\phi(x, y), m_{1}, m_{2}, \sigma_{1}, \sigma_{2}\right)
$$

$$
=\int_{\Omega}\left(\log (\sqrt{2 \pi})+\log \left(\sigma_{1}\right)+\frac{\left(S(x, y)-m_{1}\right)^{2}}{2 \sigma_{1}^{2}}\right)
$$

$$
\cdot H(\phi(x, y)) d x d y
$$

$$
+\int_{\Omega}\left(\log (\sqrt{2 \pi})+\log \left(\sigma_{2}\right)+\frac{\left(S(x, y)-m_{2}\right)^{2}}{2 \sigma_{2}^{2}}\right)
$$

$\cdot(1-H(\phi(x, y))) d x d y$. 
Means $m_{1}, m_{2}$ and variances $\sigma_{1}^{2}, \sigma_{2}^{2}$ of $S(x, y)$, respectively, inside and outside the contour " $C$ " can be calculated as follows:

$$
\begin{aligned}
m_{1} & =\frac{\int_{\Omega} S(x, y) \cdot H(\phi(x, y)) d x d y}{\int_{\Omega} H(\phi(x, y)) d x d y} \sigma_{1}^{2} \\
& =\frac{\int_{\Omega}\left(S(x, y)-m_{1}\right)^{2} \cdot H(\phi(x, y)) d x d y}{\int_{\Omega} H(\phi(x, y)) d x d y}, \\
m_{2} & =\frac{\int_{\Omega} S(x, y) \cdot(1-H(\phi(x, y))) d x d y}{\int_{\Omega}(1-H(\phi(x, y))) d x d y} \sigma_{2}^{2} \\
& =\frac{\int_{\Omega}\left(S(x, y)-m_{2}\right)^{2} \cdot(1-H(\phi(x, y))) d x d y}{\int_{\Omega}(1-H(\phi(x, y))) d x d y} .
\end{aligned}
$$

$E_{2}(\phi)$ is a regularization energy added to keep a smooth contour of the segmented region so we should minimize the length of the contour which can be calculated by the following relation:

$$
E_{2}(\phi(x, y))=\int_{\Omega}|\nabla H(\phi(x, y))| d x d y
$$

When evolving level set function, usually it may be too steep or flat so we add a third energy term $E_{3}(\phi)$ to reinitialize the level set function and to maintain the signed distance property. We use a distance regularized level set evolution (DRLSE) proposed by Wang et al. [24]:

$$
E_{3}(\phi(x, y))=\int_{\Omega} P(|\nabla \phi(x, y)|) d x d y
$$

where $P$ is called a double well potential function defined by

$$
P(z)= \begin{cases}\frac{1}{2 \pi^{2}}(1-\cos (2 \pi z)), & \text { if } z<1 \\ \frac{1}{2}(z-1), & \text { if } z>1 .\end{cases}
$$

As a result, the total energy function $E(\phi)$ can be written as

$$
\begin{aligned}
E\left(\phi, m_{1}, m_{2}, \sigma_{1}, \sigma_{2}\right)= & \alpha E_{1}\left(\phi, m_{1}, m_{2}, \sigma_{1}, \sigma_{2}\right) \\
& +\beta E_{2}(\phi)+\gamma E_{3}(\phi) .
\end{aligned}
$$

We have added $\alpha, \beta, \gamma$ as controlling parameters. We note that the parameter $\alpha$ controls the signed distance property of the level set function and the parameter $\beta$ governs its smoothness. The parameter $\gamma$ permits attracting the level set function to defect regions.

Now we keep all the variables fixed except for $\phi$; minimization of the total energy $E(\phi)$ with respect to $\phi$ is equivalent to solving the gradient descent flow equation:

$$
\frac{\partial \phi}{\partial t}=-\frac{\partial E(\phi)}{\partial \phi}
$$

We obtain the following evolution equation:

$$
\begin{aligned}
\frac{\partial \phi}{\partial t}= & \alpha \operatorname{div}(d p(|\nabla \phi|) \nabla \phi) \beta \delta(\phi) \operatorname{div}\left(\frac{\nabla \phi}{|\nabla \phi|}\right) \\
& +\gamma \delta(\phi)\left(F_{1}-F_{2}\right) .
\end{aligned}
$$

$\delta(\phi)$ is the Dirac function. $F_{1}, F_{2}, d p$ are defined as follows:

$$
\begin{aligned}
F_{i} & =\log \left(\sigma_{i}\right)+\frac{\left(S(x, y)-m_{i}\right)^{2}}{2 \sigma_{i}^{2}} \quad i=1,2, \\
d p(z) & =\frac{P^{\prime}(z)}{z} .
\end{aligned}
$$

The level set evolution equation (17) is composed of 3 terms; the first term in the right side allows keeping the regularity of the level set function, the second term preserves a smooth contour, and the third term segments the image into defect and background.

Using a simple finite difference explicit scheme to (17) we obtain the discrete level set formulation as follows:

$$
\begin{aligned}
& \phi^{n+1}=\phi^{n}+\Delta t\left[\alpha \operatorname{div}\left(d p\left(\left|\nabla \phi^{n}\right|\right) \nabla \phi^{n}\right)\right. \\
& \left.+\beta \delta\left(\phi^{n}\right) \operatorname{div}\left(\frac{\nabla \phi^{n}}{\left|\nabla \phi^{n}\right|}\right)-\gamma \delta\left(\phi^{n}\right)\left(F_{1}-F_{2}\right)\right],
\end{aligned}
$$

where $n, \Delta t$ are the index iteration number and time step, respectively.

The main stages of the proposed scheme for segmenting defects can be summarized as follows.

Step 1. Input image.

Step 2. Select a region of interest (ROI).

Step 3. Initialize parameters $\Delta t, n, \alpha, \beta, \gamma$.

Step 4. Compute off-center saliency map $S(x, y)$.

Step 5. Compute the initial level set mask $\phi_{0}$.

Step 6.

For a fixed number of iterations $n$.

Compute average and variance $m_{1}, m_{2}, \sigma_{1}, \sigma_{2}$.

Update level set function $\phi$ with (19).

Step 7. Keep the zero level of the function $\phi$.

\section{Experimental Results}

In this section the proposed level set active contour model is tested and evaluated on a set of radiography images of weld joints obtained from Federal institute of Material Research and Testing (Bam) [25]. The radiograph films have been scanned with the scanner LS85 SDR from Lumisys, mostly 


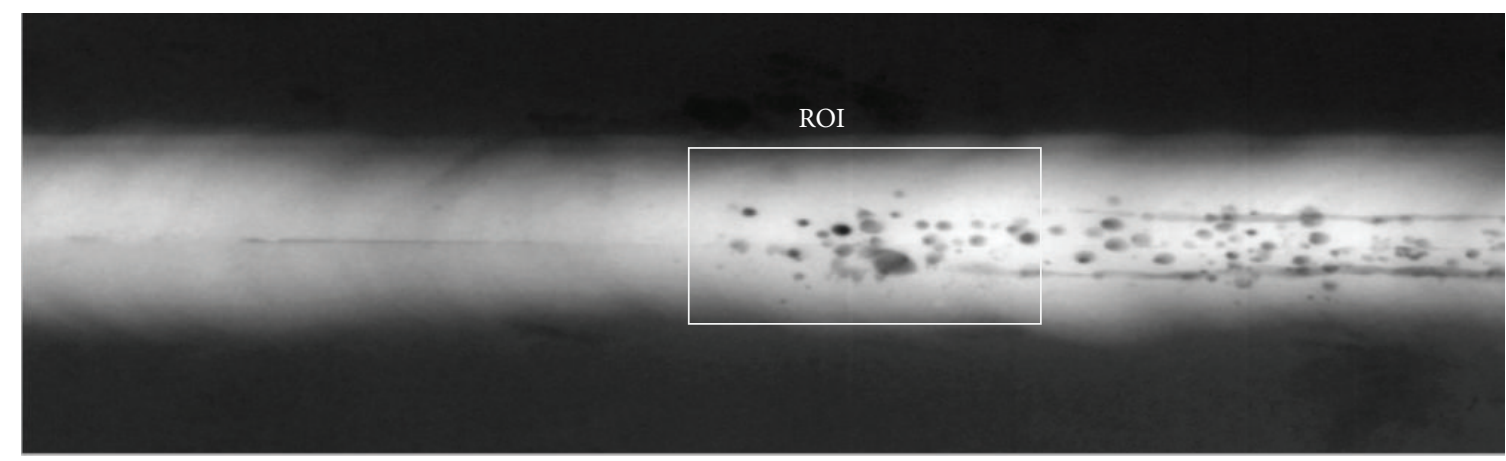

(a)

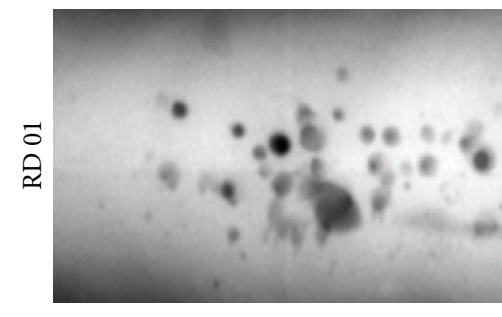

(b)

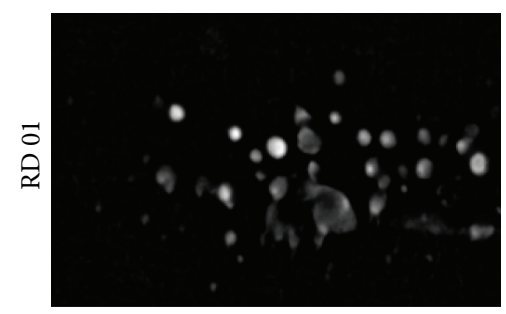

(c)

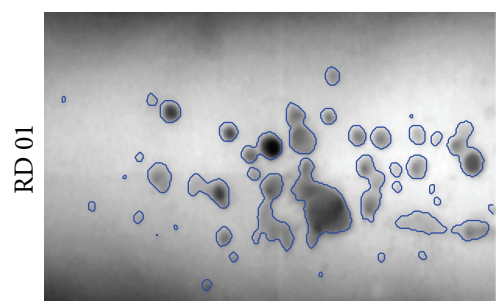

(d)

FIGURE 4: Weld defect detection with the proposed active contour: (a) the whole weld radiogram, (b) ROI selected image, (c) off-center saliency map, and (d) final segmentation with proposed level set active contour (blue color).

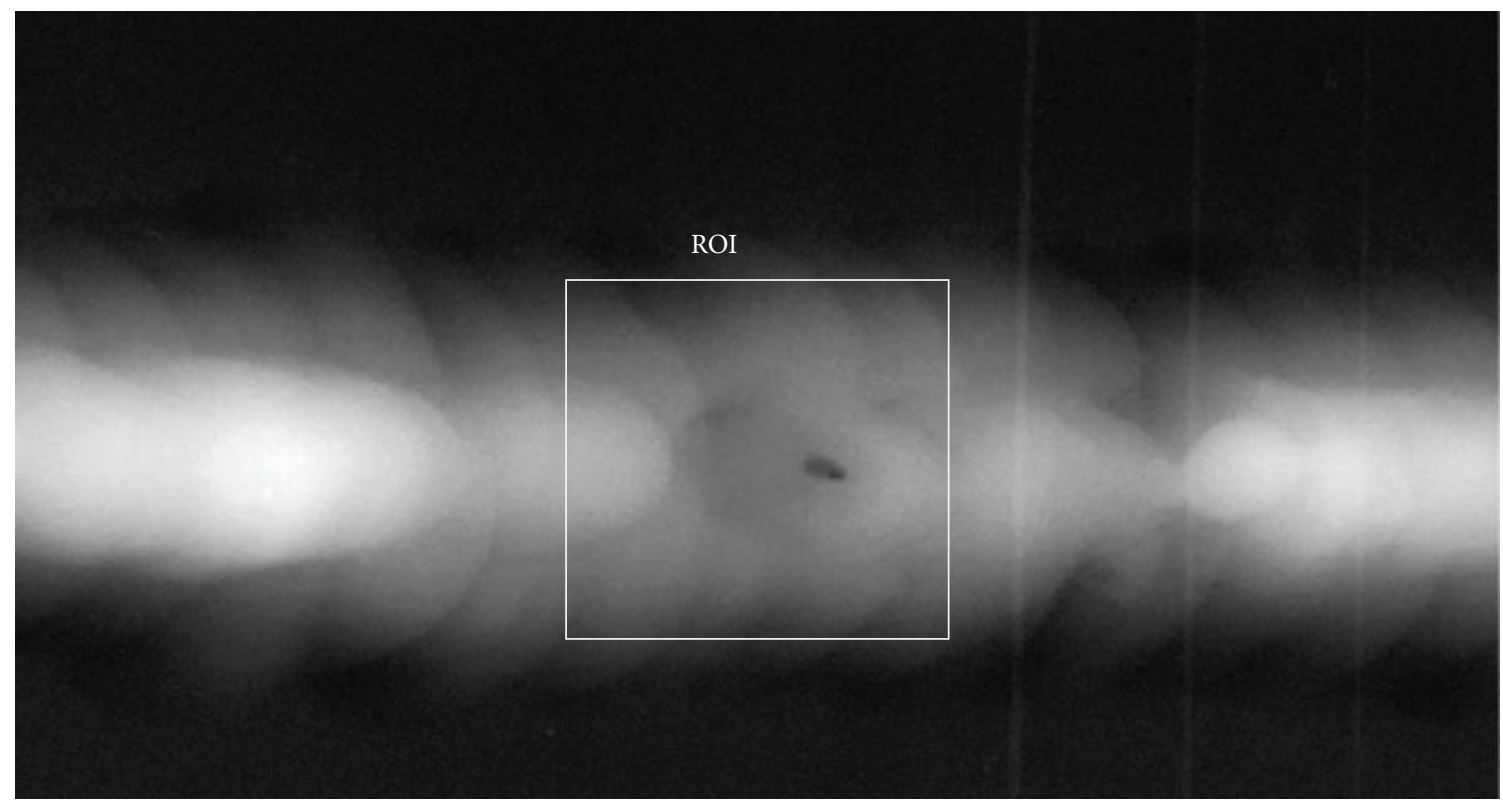

(a)

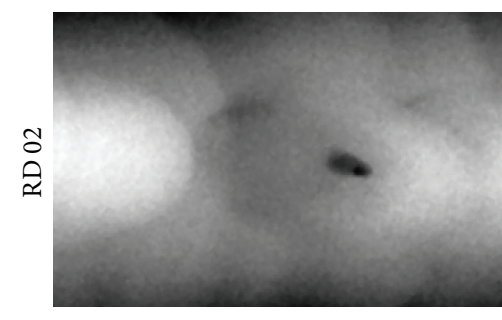

(b)

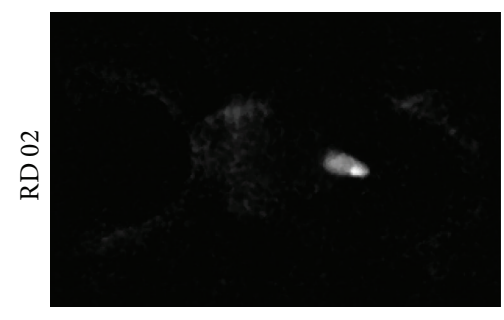

(c)

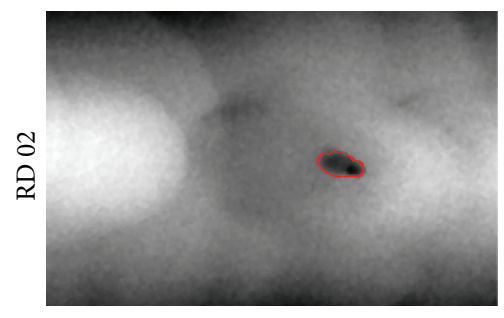

(d)

FIGURE 5: Weld defect detection with the proposed active contour: (a) the whole weld radiogram, (b) ROI selected image, (c) off-center saliency map, and (d) final segmentation with proposed level set active contour (red color). 

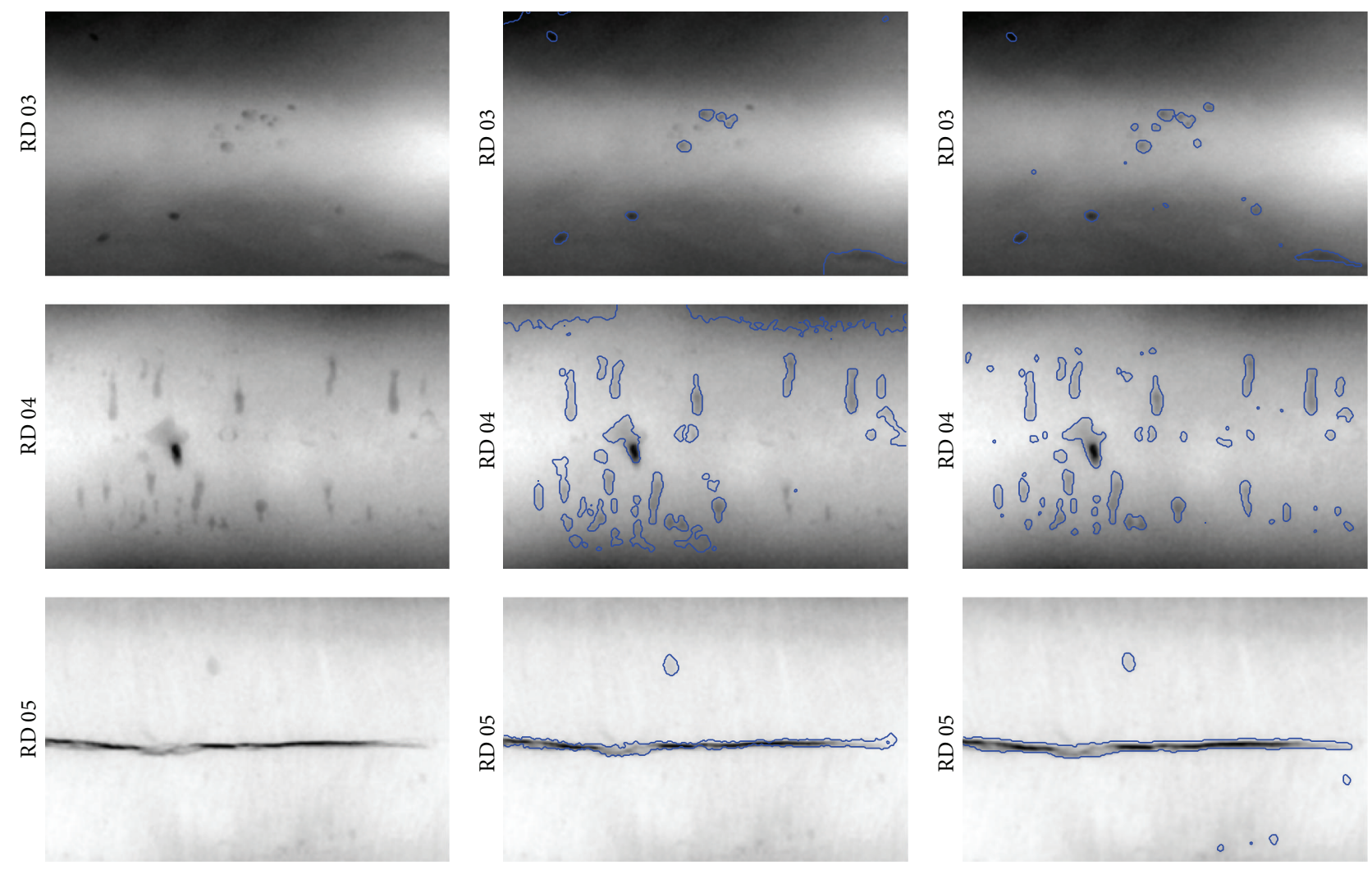

(a)

(b)

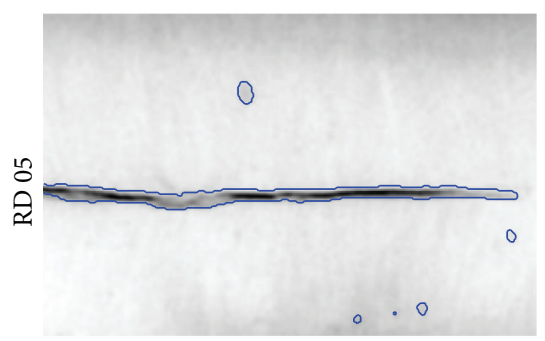

(c)

FIGURE 6: Comparative defect detection tests: (a) ROI selected image, (b) result of LIF active contour, and (c) result of the proposed method.

in high density mode. The original 12-bit data depth was rescaled to 8 bits with a linear LUT proportional to optical film density by visual adjustment to the image content. The pixel size is 40.3 micron $(630 \mathrm{dpi})$, and the images are 8bit gray values. Due to the big size of radiography weld images, the nonuniform illumination, and the small defect shape, it is difficult to detect the presence of small defects and determine accurately their sizes during the radiogram visualization. Consequently, for the seeking of simplifying the task, one could begin by selecting the region of interest, ROI, which can be considered as the parts of the image where the radiograph interpreters suspect the presence of imperfections. The selection of the ROI prevents the operator to make treatments on the irrelevant regions of the image. Moreover, it allows reducing the computing time for realtime applications noting that the technique of ROI localization is commonly used by researchers in several works. After the selection of an ROI region, the obtained images are resized to $250 \times 350$.

We present in Figures 4 and 5 the main steps of the proposed segmentation algorithm on two weld radiography examples. First row corresponds to the whole weld radiogram then a region of interest (ROI) is selected. Next images in Figures 4(b) and 5(b) show the selected region to process including various defects like porosities and slags distributed at the weld middle. The off-center saliency map is shown in the next Figures 4(c) and 5(c), as we can see defects are enhanced and the background is suppressed. Figures 4(d) and 5(d) depict the defect contour detection with a blue and red colour obtained with the proposed off-center saliency level set active contour. Even though the low contrast and the nonuniform illumination, we can see that the most parts of defects are segmented with low false detections. We note that parameters of the proposed method are set as $\Delta t=0.1$, $\alpha=0.04, \beta=0.001 \times 255 \times 255, \gamma=15$, and $n=30$.

3.1. Comparative Test 1. We show a comparison between the proposed model and a level set active contour depending only on gray level image intensity proposed by Zhang et al. [17] and called the local image fitting active contour LIF. LIF model Matlab implementation is given in author's website (http://kaihuazhang.net/J_papers/PR_10.rar). Figure 6(b) presents results of the local fitting model LIF and the results of the proposed active contour model are shown in Figure 6(c). Parameters of the proposed algorithm are fixed as $\Delta t=0.1$, $\alpha=0.02, \beta=0.001 \times 255 \times 255, \gamma=10$, and $n=30$.

Through a visual evaluation, the superiority of the proposed level set active contour can be verified. Local fitting active contour LIF [17] fails to obtain satisfactory results due to the influence of the clutter in the background and the low contrast of defects. Much false detection and a lot of defects are not detected. The best results are obtained with the proposed level set active contour. The most parts of defects are identified with low false detections. A quantitative 

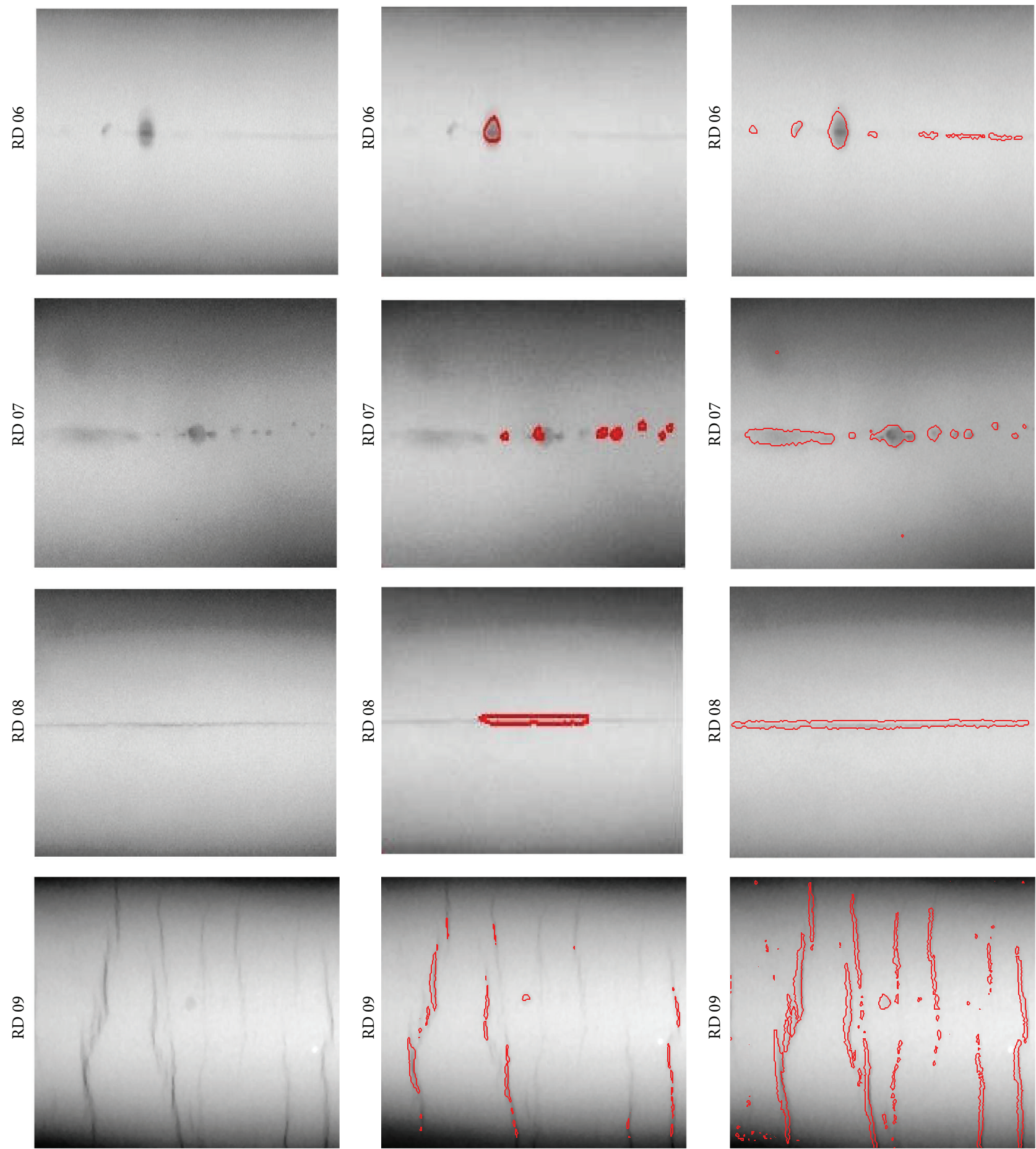

(a)

(b)

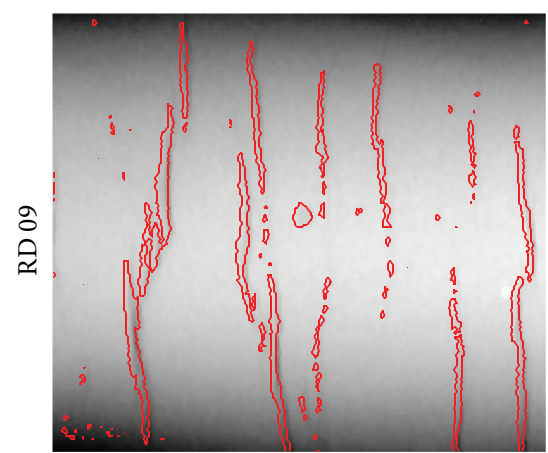

(c)

FIGURE 7: Comparative defect segmentation tests: (a) ROI selected image, (b) results of Song method, and (c) results of the proposed method.

comparison between the proposed active contour and LIF method is shown in the Table 1 . We compute a segmentation evaluation measure used by many researchers called the $F$ measure [26]. To compute this evaluation criterion we need ideal image segmentation and the proposed segmentation results; the two results should be given in binary masks. Note that ground truth or ideal segmentations used are obtained from expert visual interpretation. We note also that a higher value $(\max 1)$ denotes accurate segmentation. The detection rates are shown in Table 1 which demonstrates the high performance of our method comparing with LIF approach. Moreover, the computation time (cpu-time) is computed for two methods. The step time algorithm is fixed to 0.1 for the two methods. Algorithms are implemented on Matlab 
TABLE 1: Evaluation of segmentation accuracy and computation time.

\begin{tabular}{lcccc}
\hline & \multicolumn{2}{c}{ F-measure } & \multicolumn{2}{c}{ Cpu-Time (sec) } \\
& LIF & Prop method & LIF & Prop method \\
\hline RD 02 & 0.52 & 0.76 & 35.4 & 4.1 \\
RD 03 & 0.42 & 0.81 & 25.4 & 3.8 \\
RD 04 & 0.62 & 0.87 & 18.6 & 3.5 \\
\hline
\end{tabular}

TABLE 2: Evaluation of cpu-time convergence.

\begin{tabular}{lcc}
\hline & \multicolumn{2}{c}{ Cpu-time (sec) } \\
& Song & Prop method \\
\hline RD 06 & 8.26 & 3.85 \\
RD 07 & 9.41 & 4.81 \\
RD 08 & 9.67 & 5.23 \\
RD 09 & 9.66 & 4.77 \\
\hline
\end{tabular}

R2010a (on a PC Core i5 2.9 GHz). Obtained values show that our proposed model is very fast compared with local active contour LIF.

3.2. Comparative Test 2. We show in this paragraph a comparative test between the proposed method and saliency convex active contour model proposed by Song and Yan [18] known as SCACM. Images in Figure 7(a) corresponds to regions of interest (ROI) selected from different weld radiogram films containing various kinds of defects like porosities and horizontal and vertical thin cracks. Figure 7(b) presents SCACM method detection results and Figure 7(c) shows the segmentation results with the proposed method.

Obtained results in Figure 7 show that Song method detects only defects with small and round shapes due to the influence of the local window size used in this method. In the other side, our method does not need to use a local window as is explained before; we use a global Gaussian distribution approximation of the off-center saliency intensities. The proposed method detects defects with various forms and sizes with low false detections. Most parts of defects are extracted despite their low contrasts. Using Song method many low contrasted defects are missed and not identified. As in previous experiment, the convergence time of two methods evaluated in Table 2 shows that proposed model achieves defect segmentation in a fast time comparing with Song method. We choose parameters of the proposed algorithm as $\alpha=0.01, \beta=0.001 \times 255 \times 255, \gamma=13$, and $n=30$. We note also that we have used the Matlab implementation given by SCACM authors in their website (http://faculty.neu.edu.cn/ yunhyan/Webpage\%20for\%20article/SCACM/DemoSCACM .rar).

\section{Conclusion}

Detection of small defects in low contrasted radiography images corrupted with uneven illumination is very complicated. Usually proposed methods in this area have limited results. In this work our aim is to improve robustness of weld defect segmentation in radiography images in order to obtain satisfactory results. We have developed a new method based on level set active contour guided with an off-center saliency map. The segmentation is achieved when an energy function is minimized. Different tests on weld radiography images with various kinds of defects prove efficiency and robustness of the proposed method. A comparison test is shown with two kinds of level set active contour models. The first depends only on gray level intensity and the second uses a saliency map to derive the segmentation active contour. The comparison tests reveal that the proposed method permits overcoming the problem of nonuniform illumination and the low contrast level in radiography weld images. Moreover, our method shows a fast convergence time comparing with other methods. However, some weakness of the proposed method must be studied in a future work. To reduce human interaction, it is recommended to develop a method to select automatically the region of the interest. It is also interesting to validate this segmentation method to another radiography image application like medical area.

\section{Conflict of Interests}

The authors declare that there is no conflict of interests regarding the publication of this paper.

\section{References}

[1] I. Valavanis and D. Kosmopoulos, "Multiclass defect detection and classification in weld radiographic images using geometric and texture features," Expert Systems with Applications, vol. 37, no. 12, pp. 7606-7614, 2010.

[2] J. Zapata, R. Vilar, and R. Ruiz, "Performance evaluation of an automatic inspection system of weld defects in radiographic images based on neuro-classifiers," Expert Systems with Applications, vol. 38, no. 7, pp. 8812-8824, 2011.

[3] C. Stolojescu-Crişan and Ş. Holban, "A comparison of X-ray image segmentation techniques," Advances in Electrical and Computer Engineering, vol. 13, no. 3, pp. 85-92, 2013.

[4] A. Mahmoudi and F. Regragui, "Welding defect detection by segmentation of radiographic images," in Proceedings of the World Congress on Computer Science and Information Engineering (WRI'09), vol. 7, pp. 111-115, Los Angeles, Calif, USA, March 2009.

[5] D. Mery and M. A. Berti, "Automatic detection of welding defects using texture features," Insight: Non-Destructive Testing and Condition Monitoring, vol. 45, no. 10, pp. 676-681, 2003.

[6] M. A. Carrasco and D. Mery, "Segmentation of welding defects using a robust algorithm," Materials Evaluation, vol. 62, no. 11, pp. 1142-1147, 2004.

[7] E. S. Amin, "Application of artificial neural networks to evaluate weld defects of nuclear components," Journal of Nuclear and Radiation Physics, vol. 3, no. 2, pp. 83-92, 2008.

[8] S.-B. Zhou, A.-Q. Shen, and G.-F. Li, "Concrete image segmentation based on multiscale mathematic morphology operators and Otsu method," Advances in Materials Science and Engineering, vol. 2015, Article ID 208473, 11 pages, 2015.

[9] S. Osher and N. Paragios, Geometric Level Set Methods in Imaging, Vision, and Graphics, Springer, 2003. 
[10] X.-F. Wang, D.-S. Huang, and H. Xu, "An efficient local ChanVese model for image segmentation," Pattern Recognition, vol. 43, no. 3, pp. 603-618, 2010.

[11] C. Xu and J. L. Prince, "Snakes, shapes, and gradient vector flow," IEEE Transactions on Image Processing, vol. 7, no. 3, pp. 359-369, 1998.

[12] T. F. Chan and L. A. Vese, "Active contours without edges," IEEE Transactions on Image Processing, vol. 10, no. 2, pp. 266-277, 2001.

[13] D. Mumford and J. Shah, "Optimal approximations by piecewise smooth functions and associated variational problems," Communications on Pure and Applied Mathematics, vol. 42, no. 5, pp. 577-685, 1989.

[14] L. Wang, C. Li, Q. Sun, D. Xia, and C.-Y. Kao, "Active contours driven by local and global intensity fitting energy with application to brain MR image segmentation," Computerized Medical Imaging and Graphics, vol. 33, no. 7, pp. 520-531, 2009.

[15] X. Liu, S.-J. Peng, Y.-M. Cheung, Y. Y. Tang, and J.-X. Du, “Active contours with a joint and region-scalable distribution metric for interactive natural image segmentation," IET Image Processing, vol. 8, no. 12, pp. 824-832, 2014.

[16] K. Zhang, L. Zhang, H. Song, and W. Zhou, "Active contours with selective local or global segmentation: a new formulation and level set method," Image and Vision Computing, vol. 28, no. 4, pp. 668-676, 2010.

[17] K. Zhang, H. Song, and L. Zhang, "Active contours driven by local image fitting energy," Pattern Recognition, vol. 43, no. 4, pp. 1199-1206, 2010.

[18] K. Song and Y. Yan, "Micro surface defect detection method for silicon steel strip based on saliency convex active contour model," Mathematical Problems in Engineering, vol. 2013, Article ID 429094, 13 pages, 2013.

[19] L. Itti and C. Koch, "Computational modelling of visual attention," Nature Reviews Neuroscience, vol. 2, no. 3, pp. 194-203, 2001.

[20] R. Achantay, S. Hemamiz, F. Estraday, and S. Süsstrunky, "Frequency-tuned salient region detection," in Proceedings of the IEEE Computer Society Conference on Computer Vision and Pattern Recognition (CVPR '09), pp. 1597-1604, IEEE, June 2009.

[21] X. Hou and L. Zhang, "Saliency detection: a spectral residual approach," in Proceedings of the IEEE Conference on Computer Vision and Pattern Recognition (CVPR '07), pp. 1-8, Minneapolis, Minn, USA, June 2007.

[22] S. Montabone and A. Soto, "Human detection using a mobile platform and novel features derived from a visual saliency mechanism," Image and Vision Computing, vol. 28, no. 3, pp. 391-402, 2010.

[23] P. Viola and M. J. Jones, "Robust real-time face detection," International Journal of Computer Vision, vol. 57, no. 2, pp. 137154, 2004.

[24] L. Wang, L. He, A. Mishra, and C. Li, "Active contours driven by local Gaussian distribution fitting energy," Signal Processing, vol. 89, no. 12, pp. 2435-2447, 2009.

[25] http://www.bam.de/en/index.htm.

[26] https://en.wikipedia.org/wiki/Precision_and_recall. 

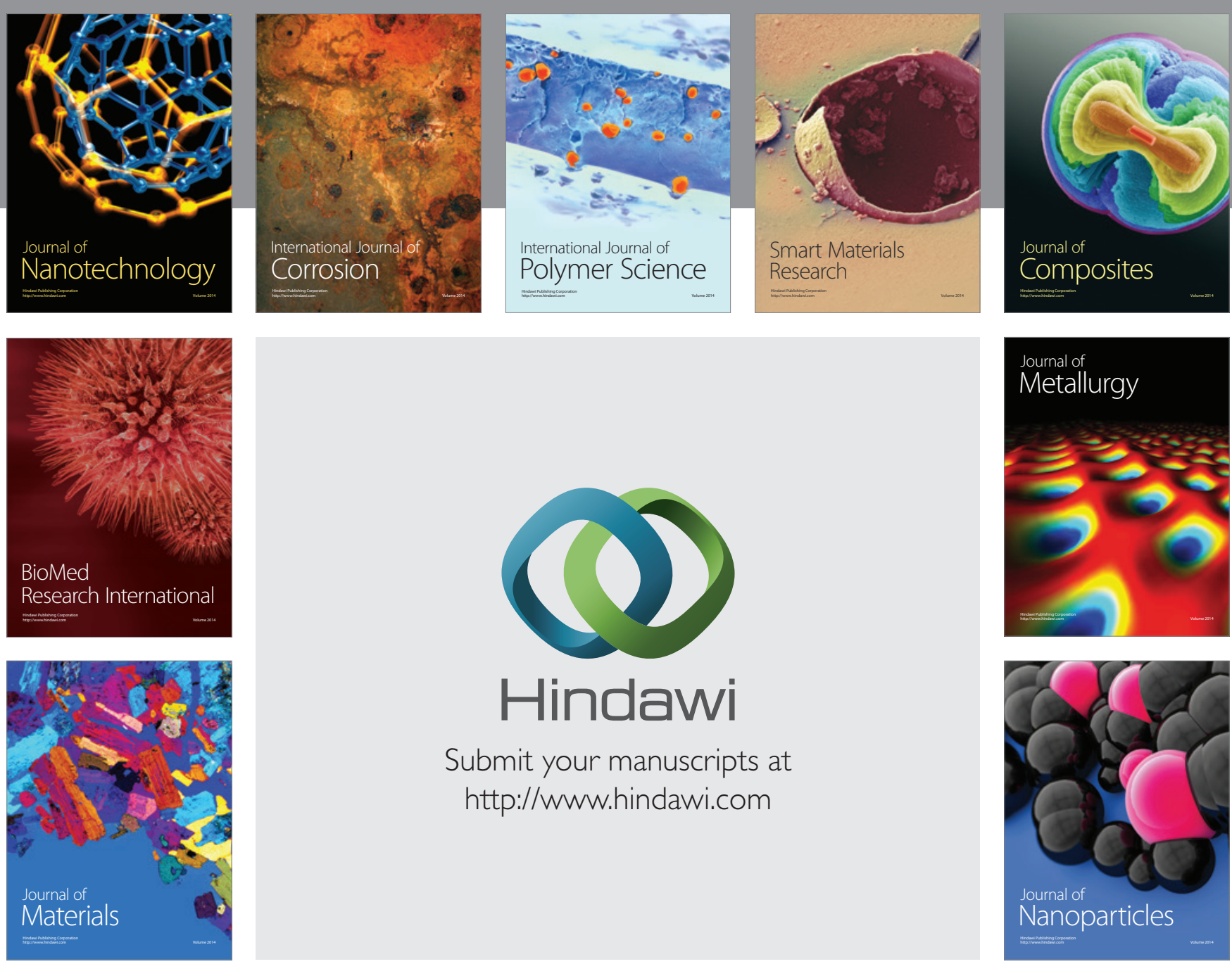

Submit your manuscripts at http://www.hindawi.com
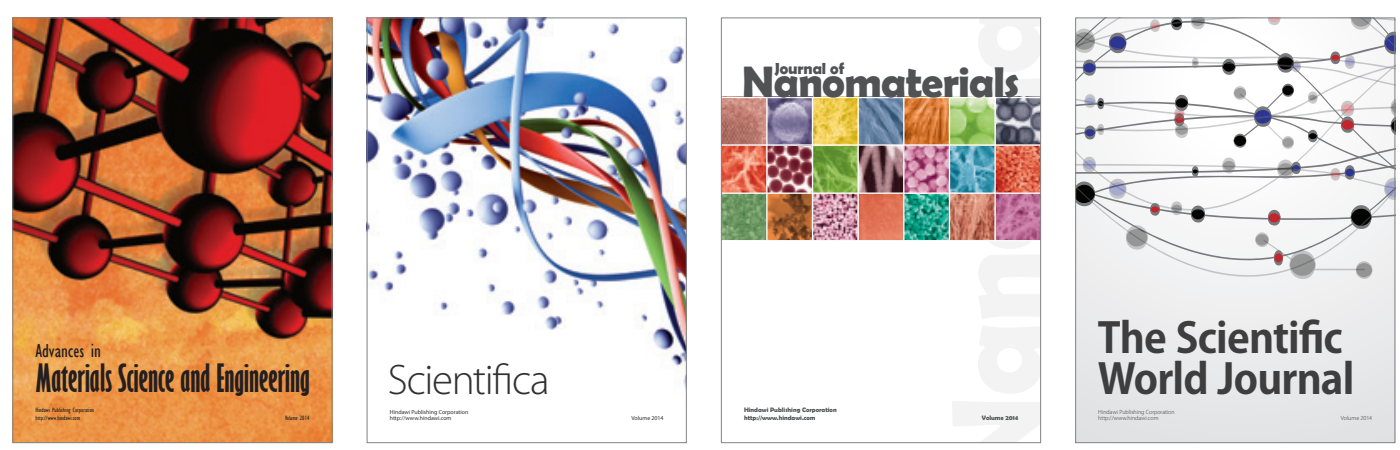

\section{The Scientific World Journal}
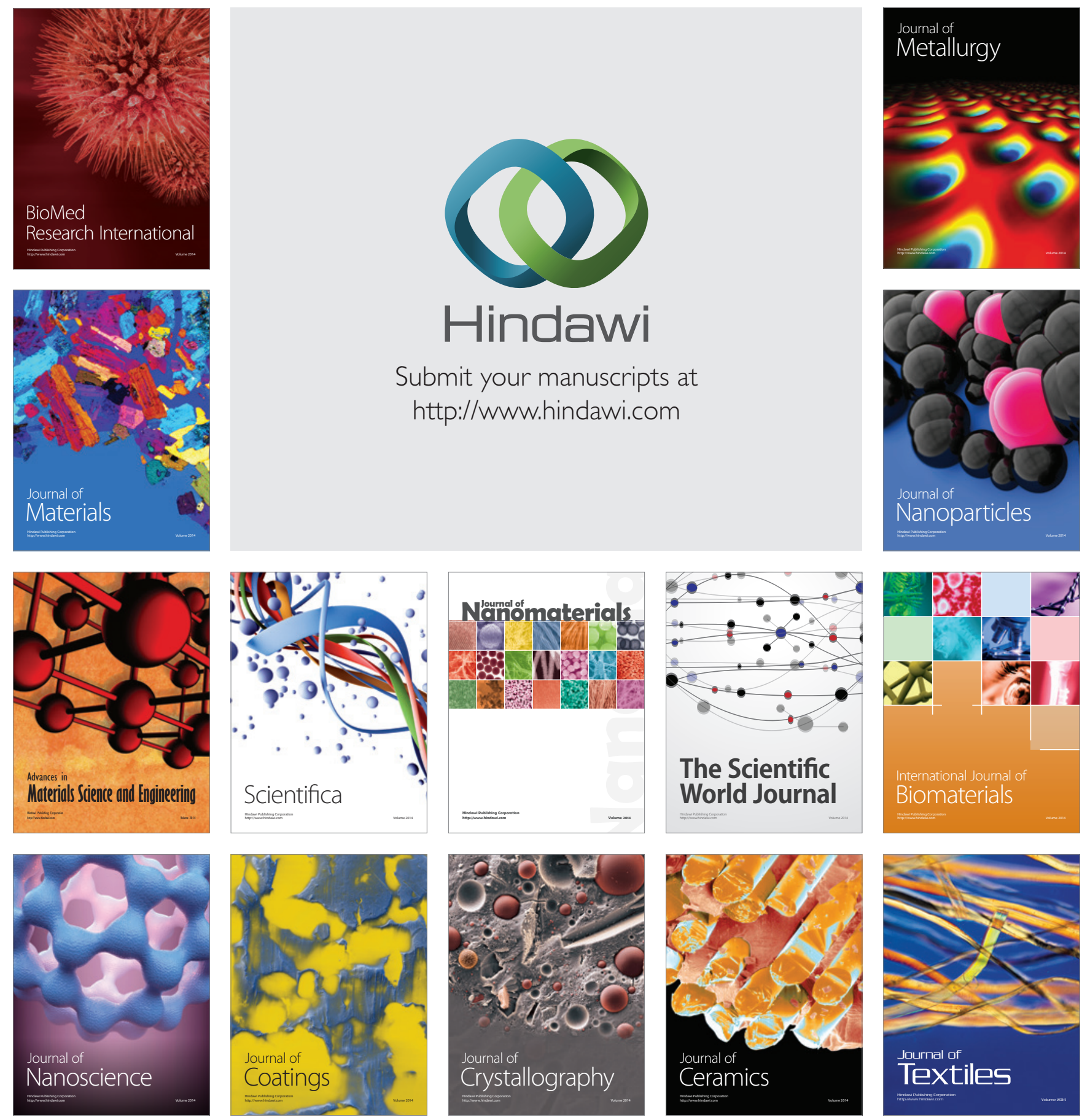